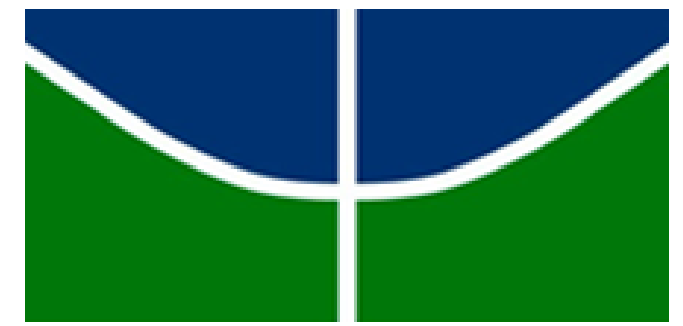

Universidade de Brasília

Faculdade de Ciências da Saúde

Departamento de Saúde Coletiva

DIANE CARLOS DE JESUS

\title{
A SAÚDE MENTAL DA POPULAÇÃO RURAL E SUA RELAÇÃO COM OS DETERMINANTES SOCIAIS E AS INIQUIDADES DE SAÚDE: UMA REVISÃO DE LITERATURA
}

Brasília

2019 
DIANE CARLOS DE JESUS

\section{A SAÚDE MENTAL DA POPULAÇÃO RURAL E SUA RELAÇÃO COM OS DETERMINANTES SOCIAIS E AS INIQUIDADES DE SAÚDE: UMA REVISÃO DE LITERATURA}
Trabalho de Conclusão de Curso (TCC) de graduação apresentado ao Departamento de Saúde Coletiva como requisito parcial para obtenção do título de Bacharela em Saúde Coletiva.

Orientador: Muna Muhammad Odeh

Brasília

2019 
Diane Carlos de Jesus

\section{A SAÚDE MENTAL DA POPULAÇÃO RURAL E SUA RELAÇÃO COM OS DETERMINANTES SOCIAIS E AS INIQUIDADES DE SAÚDE: UMA REVISÃO DE LITERATURA}

Trabalho de Conclusão de Curso (TCC) apresentada à disciplina de TCC, do curso de Saúde Coletiva da Universidade de Brasília (UnB), como requisito parcial à obtenção de título de Bacharel em Saúde Coletiva.

ORIENTADORA: Prof ${ }^{\mathrm{a}}$ Muna Muhammad Odeh

\section{BANCA EXAMINADORA}

Enf. Jaqueline Nicácio

Gerente de Serviço de Atenção Primária 2 do Paranoá

Secretaria de Saúde do Distrito Federal

Brasília

2019 


\section{RESUMO}

A população rural vive em uma situação considerada de vulnerabilidade, devido à influência de fatores socioeconômicos, culturais, ambientais políticos etc. Esses fatores associados às iniquidades de saúde e aos determinantes sociais remontam um cenário na qual é observável uma situação de saúde mental bastante preocupante, delicada e complexa. Objetivo: Analisar a saúde mental da população rural e suas relações com os determinantes sociais de saúde e as iniquidades em saúde, de modo a evidenciar os fatores que não só colaboram, mas também interferem e agravam a situação de saúde mental da população rural. Utilizou-se do método de revisão de literatura do tipo revisão narrativa, com base em base de dados e periódicos como SciELO, LILACS, Redalyc e Pubmed. Observa-se que a população rural é vítima do esquecimento por parte de políticas públicas diversas, inclusive na área da saúde. Diante disso, nota-se uma necessidade urgente do desenvolvimento de políticas e estratégias voltadas à saúde mental dessa população, observando suas particularidades especificidades, de modo a reduzir as iniquidades em saúde.

Palavras chaves: saúde mental; população rural; determinantes sociais da saúde; iniquidades em saúde. 


\begin{abstract}
The rural population lives in a situation considered of vulnerability, due to the influence of socioeconomic, cultural, environmental and political factors, etc. These factors associated with health inequities and social determinants go back to a scenario in which a very worrying, delicate and complex mental health situation is observable. Objective: To analyze the mental health of the rural population and their relationship with the social determinants of health and health inequities, in order to highlight the factors that not only collaborate, but also interfere and aggravate the mental health situation of the rural population. The literature review method was used as a narrative review, based on data bases and periodicals such as SciELO, LILACS, Redalyc and Pubmed. It is observed that the rural population is a victim of forgetfulness by diverse public policies, including in the area of health. Given this, there is an urgent need for the development of policies and strategies aimed at the mental health of this population, observing their particularities specificities, in order to reduce health inequities.
\end{abstract}

Keywords: Mental health; rural population; social determinants of health; inequities in health. 
SUMÁRIO

1. INTRODUÇÃO

1.1 Saúde Mental 6

$\begin{array}{ll}1.2 \text { População Rural } & 7\end{array}$

1.3 Iniquidades de saúde 9

$\begin{array}{ll}\text { 1.4 Determinantes sociais de saúde } & 10\end{array}$

2. JUSTIFICATIVA 14

3. OBJETIVOS 14

4. METODOLOGIA

5. RESULTADOS E DISCUSSÃO

5.1 A saúde mental da população rural e sua estreita 15

relação com os determinantes sociais

5.2 As iniquidades em saúde e sua influência 21

na saúde mental da população rural

6.CONSIDERAÇÕES FINAIS

7. REFERÊNCIAS BIBLIOGRÁFICAS 26 


\section{INTRODUÇÃO}

\subsection{Saúde Mental}

Definir o termo "saúde mental" não é algo tão fácil, pois ele possui um significado bastante amplo, que supera a simples compreensão de ausência de perturbações mentais. Assim, tem aumentado à percepção e compreensão de que a saúde mental é o resultado de interações variadas e complexas, que envolve fatores tanto sociais quanto psicológicos, biológicos, genéticos e ambientais. (ALVES; RODRIGUES, 2010, PATEL et al., 2010). De acordo com a Organização Mundial da Saúde (WHO, 2001), nesse conceito são incluídas questões de ordem do indivíduo como autonomia, competência social, bem-estar subjetivo, auto realização do seu potencial tanto emocional quanto intelectual, auto eficácia percepcionada dentre outros. Ao considerar que esse termo é bastante subjetivo, histórico e culturalmente construído e que abarca as mais variadas perspectivas profissionais, entende-se que não há como ter apenas uma única definição de saúde mental. Muitas vezes, os próprios ambientes e lugares em que as pessoas vivem, assim como diferentes fases da vida, relacionam-se com o surgimento das doenças mentais. (WHO; Calouste Gulbenkian Foundation, 2014).

Alguns estudiosos já analisam a saúde mental sob a perspectiva do próprio contexto e da conjuntura social. Desse modo, a saúde mental também é vista e compreendida através do quanto o indivíduo é capaz de interagir com os mais variados grupos e com o próprio ambiente, a tal ponto que promova seu próprio bem-estar subjetivo e a sua habilidade afetiva, cognitiva, dentre outras habilidades mentais associadas. Tal definição nos traz a compreensão de que saúde mental não se trata apenas de algo relacionado ao plano individual, pois pode também estar relacionado com o próprio indivíduo interagindo não apenas com o ambiente, mas também, com os mais diversos grupos. (ROSA, 2007)

Ademais, como os transtornos mentais atualmente são uma das principais causas de morbidade, a temática saúde mental tornou-se o centro de um debate sobre iniquidades e desigualdades em saúde, uma vez que esses transtornos 
impactam significativamente a vida tanto dos indivíduos quanto de seus familiares. Além disso, a relação entre quem de fato possui um sofrimento mental e aqueles que recebem cuidados e tratamento é considerada bastante desproporcional. (OMS, 2009). Observa-se, portanto que é de suma importância discutir a questão da saúde mental também como modo de se buscar preservar cada vez mais, a qualidade de vida dos indivíduos. (OMS, 2005)

Isto posto, ressalta-se que para que haja efetivamente, melhorias no âmbito da saúde mental e também uma diminuição global relacionado às doenças mentais faz-se necessário conhecer de fato os determinantes econômicos e sociais que estão intrinsecamente relacionados à saúde mental de uma população, segundo tem estabelecido na literatura.(ALVES; RODRIGUES, 2010).

\subsection{A população rural}

Com base nos dados do Censo demográfico de 2010, havia no Brasil, 190.755.799 habitantes, sendo que 15,6\% moravam em zonas rurais. É na região centro-oeste que se encontra a menor população rural, enquanto a maior, está situada no nordeste. Em 1960, notou-se que a população rural, com um índice de $54,9 \%$, era maior do que a urbana. Contudo, a cada censo realizado essa população reduzia-se em todo o Brasil. Com isso, é notório esse êxodo rural contínuo,

principalmente das mulheres jovens, filhas de agricultores, que buscam outras profissões no meio urbano. (SOARES et al., 2015).

Houve então, uma diminuição de residentes em domicílios rurais do sexo feminino, cujos percentuais apresentados são inferiores ao masculino na série histórica dos censos de 1970-2010, enquanto no perfil urbano do país, ocorreu o contrário. Assim, no ano de 2010, a população masculina já correspondia a 53\% 6, sendo que a agricultura mecanizada e a pecuária extensiva contribuíram para essa masculinização, enquanto a agricultura familiar contribuiu para a permanência das mulheres no meio rural. (SOARES et al., 2015). 
A população rural vive em condições caracterizadas por determinantes socioeconômicas e índices de saúde cujo impacto é maior e mais devastador do que em populações urbanas. Além disso, alguns problemas de saúde, como doenças típicas daquele meio, exposições a condições ambientais desfavoráveis a saúde e acidentes ocorrem com mais frequência nas zonas rurais. Os índices de cobertura no âmbito da prevenção são bem piores nessas aéreas também. Para essa população, o acesso a serviços de saúde em todos os níveis, geralmente é mais difícil, assim como o acesso a longitudinalidade do cuidado. Algo também observado tanto no Brasil quanto no mundo todo, é a falta de recursos humanos no âmbito rural, inclusive profissionais de saúde para ações de assistência e de promoção. (ANDO et al., 2011).

Diante desse quadro, observa-se a importância do reconhecimento de que essas especificidades e particularidades vivenciadas no âmbito rural interferem diretamente nos modos de vida, de subjetivação e construção das identidades sociais, bem como remodelam frequentemente, o cenário social (ANDO et al., 2011). Assim, sob essa perspectiva, considera-se o meio rural como um espaço dinâmico, que passa por constantes transformações, de reconfigurações e engloba aspectos culturais, socioeconômicos políticos e subjetivos (SANTOS M., 1994). O meio rural, trata-se, portanto, de um território híbrido, onde há uma propagação de hábitos alimentares, modos de trabalho, de higiene, de relações sociais, exercício da sexualidade, de adoecimento e cuidado com a saúde, que se relacionam diretamente, com as condições de vida, tradições, valores, e códigos morais presentes nesse território, algo que também se aplica à saúde mental (VELOSO et al., 2016).

O espaço rural, além de ser um ambiente de vida e trabalho, ele é também uma paisagem cultural e ecológica que produz específicas representações de pertencimento, de relações sociais, desejos e projetos de vida. Ele deixou de ser um lugar homogêneo, contendo apenas paisagens e personagens das regiões, ao abarcar novos atores e meios de produção, através do processo de modernização 
da agricultura e da articulação com a cidade, juntamente com suas práticas e saberes (FERREIRA, 2002).

Embora haja a permanência da pobreza no meio rural, nas últimas décadas houve um crescimento da renda e redução das desigualdades sociais no meio, rural (HELFAND; ROCHA; VINHAIS, 2009). Contudo, as melhorias nas condições de vida dessa população, que ocorreram devido às políticas agrárias, foram bastante incipientes, e apenas atenuaram questões relativas a conflitos sociais, ao invés de modificar de fato, as condições de desigualdade social e pobreza. (DIMENSTEIN et al., 2014).

Diante disso, percebe-se que para o aumento da qualidade de vida na zona rural, evitando assim as desigualdades sociais e êxodo rural, faz se necessário investir em melhorias contínuas dos serviços prestados, por meio de políticas públicas adaptadas ao contexto rural (FLORIANO, 2009).

\subsection{Iniquidades em saúde}

Embora nos últimos anos tenha havido melhorias relacionadas aos indicadores de saúde no Brasil, ele ainda encontra-se entre os países com maiores índices de iniquidades em saúde, que são as desigualdades entre indivíduos e grupos populacionais, e que apesar de relevantes e sistemáticas, são injustas desnecessárias e evitáveis (WHITEHEAD, 1992). As iniquidades em saúde são um dos aspectos considerados mais marcantes no âmbito da situação de saúde no Brasil.

As iniquidades em saúde são oriundas das desigualdades bastante grandes e marcantes entre os vários estratos econômicos e sociais da população brasileira. Com base no relatório do programa das Nações Unidas de 2007, analisando dados de 2005, o Brasil ocupa o décimo primeiro lugar no ranking entre países com as maiores desigualdades no mundo no que se refere à distribuição de renda (CNDSS, 2008). 
Aumentaram nos últimos anos a qualidade e quantidade de estudos que abordam a relação entre as desigualdades nas condições de vida a saúde das populações e o grau de desenvolvimento da teia associações e vínculos entre indivíduos grupos. Tais estudos permitem observar que ao ser superado um determinado limite relacionado ao crescimento econômico de um país, caso haja um crescimento a mais de riqueza, não significa que isso trará melhorias relevantes nas condições de saúde. Partindo dessa percepção, nota-se que a riqueza total não é um fator suficiente para explicar a situação de saúde geral de um país, pois para se mensurar ela, o fator mais importante é o modo como se dá a distribuição dessa riqueza. (BUSS; FILHO, 2006)

Observa-se também que não é toda diferença no âmbito da situação de saúde que pode ser considerada iniquidade. Contudo, toda desigualdade ou diferença que pode ser reduzida, relacionada às condições heterogêneas de vida, são iniquidades (CASTELLANOS, 1997).

Além do âmbito prático e teórico, a discussão referente às iniquidades também envolvem uma dimensão ética. Logo, ao se tomar como injustas as diferenças relativas ao perfil epidemiológico dos mais diversos grupos sociais, o que se espera é que essa iniquidade seja reparada, e de preferência, de forma imediata. (BARATA, 2001)

Além disso, na busca pela superação das iniquidades em saúde, faz se necessário que haja uma participação e envolvimento ativo das pessoas, na política, principalmente daqueles constantemente oprimidos e cujos direitos, mesmo que fundamentais, Ihes são negados de um modo mais constante. Assim, ao reivindicar politicamente os seus direitos, essas pessoas podem não apenas conquistar a igualdade, mas também contribuir para a criação de novos direitos (CHAUÍ, 2001).

\subsection{Determinantes sociais de saúde}

Ao se tratar de saúde e qualidade de vida, um dos principais fatores considerados é os determinantes sociais. Assim, em paralelo com os fatores 
relativos a aspectos biológicos, esses determinantes sociais de saúde influenciam bastante na saúde das pessoas. Sua influência é tão grande, que atualmente estima-se que seja superior do que os próprios fatores relacionados à fatores biológicos (GEORGE, 2011; PORTRAIT; LINDEBOOM; DEEG, 2001). Desse modo, considera-se que o conjunto de fatores socioeconômicos, culturais, ambientais e biológicos, é responsável por determinar o equilíbrio do processo saúde-doença. Contudo, mesmo frente à notável influência de fatores externos na saúde dos indivíduos, tais considerações nem sempre são incluídas na formulação de políticas públicas em saúde (CARRAPATO; CORREIA; GARCIA, 2017).

Há várias definições de determinantes sociais de saúde (DDS), que expressam de uma forma mais ou até menos detalhada, o conceito de que as condições de vida e trabalho dos grupos populacionais entre indivíduos relacionamse com a sua situação de saúde (BUSS; FILHO, 2007).

Segundo a Comissão Nacional sobre os Determinantes Sociais da Saúde (CNDSS), os determinantes sociais de saúde são fatores socioeconômicos, culturais, políticos, étnico-raciais, psicológicos e comportamentais, que influem a ocorrência de fatores de risco ou problemas de saúde na população (CNDSS, 2008). Já a comissão homônima da Organização Mundial da Saúde (OMS) utiliza uma definição mais curta onde de acordo com ela, os determinantes sociais de saúde são as condições sociais nas quais a população vive ou trabalha.

Krieger (2001) utiliza uma definição de DDS na qual eles são tidos como mecanismos e fatores pelos quais as condições sociais influenciam na saúde, porém podem ser alterados potencialmente através de ações fundadas em informações e ações educacionais. Já a definição proposta por Tarlov (1996) é bem pequena e sugere que os determinantes são simplesmente, características nas quais a vida transcorre, acontece e se desenvolve dentro delas. Desse modo, percebe-se que a influência dos determinantes sobre o estado de saúde dos indivíduos, de fato é algo inquestionável (BUSS; FILHO, 2007). Além de que, mais de $50 \%$ da influência exercida sobre a saúde, dos indivíduos tem como base, as condições em que as pessoas, nascem, vivem, crescem envelhecem e trabalham. Assim, compreendendo 
tais fatores, é possível buscar estratégias de intervenção que se adequam aos vários níveis, de modo a reduzir o seu efeito prejudicial à saúde (CAMPOS; SATURNO; CARNEIRO, 2010).

Observa-se que nas últimas décadas tanto na literatura nacional quanto internacional, houve um avanço significativo em estudos sobre o modo como se desenvolve e se organiza uma determinada sociedade e também sobre sua situação de saúde (ALMEIDA-FILHO, 2003). Desse modo, há três gerações que abordam os estudos a respeito das iniquidades em saúde, sendo que a primeira buscou descrever as relações existentes entre saúde e pobreza, enquanto a segunda descreveu os gradientes de saúde conforme os diversos critérios de estratificação econômico-social, e a terceiro, que também é a geração atual, dedica-se principalmente a estudar os mecanismos e modos de produção das iniquidades (ADLER, 2006).

Em estudos que abordam a relação entre saúde e determinantes sociais um dos principais desafios existentes é o estabelecimento de uma hierarquia de determinações entre os fatores de natureza política econômica e social, e as mediações por meio das quais esses fatores, recaem sobre a situação de saúde dos indivíduos e grupos, uma vez que a relação de determinação não é algo simples e direto como uma relação de causa e efeito. Assim, conhecendo esse complexo de mediações é possível compreender, por exemplo, o porquê de não haver uma constante correlação entre indicadores de saúde e os macro indicadores de riqueza de um povo, como o produto interno bruto (PIB). Mesmo que o volume de riqueza que uma sociedade gera, seja um elemento importante para a viabilização de melhores condições de saúde e vida, o estudo dessas questões permite compreender porque há países que mesmo tendo um PIB inferior a outros países ainda assim, possuem indicadores de saúde melhores do que estes. Estudando essas mediações também é possível identificar como e onde devem ser realizadas intervenções para que elas provoquem mais impacto e desse modo reduzam as iniquidades em saúde (BUSS; PELEGRINI FILHO, 2007). 
Outro importante desafio, diz respeito à forma de diferenciar quando os determinantes estão relacionados ao plano individual, e quando eles envolvem e relacionam-se com grupos e populações, já que alguns fatores relacionados ao indivíduo e às condições de saúde das pessoas, não são suficientemente capazes de explicar as diferenças existentes entre às diversas populações e grupos e sociedades. Porém, ambos os fatores, tanto individuais quanto populacionais, possuem uma grande importância, pois enquanto um consegue identificar quais pessoas de dentro de um grupo, estão mais expostas a um risco, o outro ao comparar, analisa fatores como o quanto o grau de equidade e de distribuição de renda, entre as populações e grupos, afetam, interfere e relaciona-se com as diferenças nas condições de saúde existentes entre eles (KAWACHI et al., 1997; WILKINSON, 1997; FILHO, 2000).

Sabe-se que há diversas abordagens para o estudo dos mecanismos por meio dos quais os determinantes acarretam as iniquidades em saúde. A primeira abordagem enfatiza os aspectos físicos e materiais, na produção saúde e da doença, partindo da compreensão de que as diferenças de renda afetam e influenciam a saúde, devido à escassez de recursos por parte dos indivíduos e também pela falta de investimentos em infraestrutura comunitária, como transporte, habitação educação, serviços de saúde e saneamento. Tudo isso são resultantes das decisões políticas e processos econômicos. Há outro enfoque que prioriza os fatores psicossociais, buscando explorar as relações entre a situação de saúde, as percepções de desigualdades sociais e os mecanismos psicobiológicos, partindo da concepção de que as experiências e percepções das pessoas que vivem em sociedades desiguais geram prejuízos à saúde e estresse. Os enfoques multiníveis ou ecossociais objetivam agregar as abordagens grupais, individuais, biológicas e sociais em uma perspectiva histórica, dinâmica e ecológica. Por fim, há também os enfoques que visam analisar as relações existentes entre as condições de vida desiguais, a saúde das populações e o nível de desenvolvimento da teia de associações e vínculos entre grupos e indivíduos. Existem também, diversos modelos que procuram esquematizar de várias formas, a trama de relações e 
associações entre os diversos fatores que são estudados através desses diversos e diferentes enfoques. (BUSS; PELEGRINI FILHO, 2007).

\section{JUSTIFICATIVA}

A importância desse trabalho se justifica pelo fato de haverem poucos estudos relacionados à saúde mental da população rural, tanto em âmbito nacional quanto internacional. Além disso, percebe-se também a necessidade de se evidenciar os fatores e as possíveis causas dos acometimentos e doenças mentais, no âmbito rural, uma vez que possuem especificidades e particularidades culturais, socioambientais, econômicas e políticas que contribuem e afetam de forma incisiva, na saúde tanto física, quanto mental dessa população. Contudo, muitas políticas, programas e estratégias desenvolvidas a essa população, na área da saúde e nas demais áreas, ainda são elaboradas com base em modelos aplicados às populações urbanas.

Diante disso, observa-se que essa pesquisa também possa contribuir para o aprimoramento e desenvolvimento de estratégias, políticas e programas voltados às melhorias no âmbito da saúde mental dessa população.

\section{OBJETIVOS}

\section{Objetivo Geral}

- Evidenciar e analisar a relação entre a saúde mental da população rural com os determinantes sociais de saúde e as iniquidades de saúde, com base em revisão bibliográfica.

\section{Objetivos Específicos}

- Sintetizar estudos e pesquisas que relacionam a influência das iniquidades de saúde e dos determinantes sociais na saúde mental da população rural. 
- Sublinhar as especificidades do meio rural no que concerne seu impacto na saúde mental da população, apresentando propostas de melhoria conforme mencionado na literatura.

\section{METODOLOGIA}

Essa pesquisa trata-se de uma revisão de literatura, do tipo revisão narrativa. "Nesse tipo de estudo, são analisadas as produções bibliográficas em "determinada área [...] fornecendo o estado da arte sobre um tópico específico, evidenciando novas ideias, métodos, subtemas que têm recebido maior ou menor ênfase na literatura selecionada" (NORONHA; FERREIRA, 2000, p. 191).

Para isso, foram utilizados artigos científicos, monografias, publicações em congressos, e em bases de dados e periódicos como SciELO (Scientific Electronic Library Online), LILACS (Literatura Latino Americana e do Caribe em Ciências da Saúde), Redalyc (Rede de Revistas Científicas da América Latina e Caribe), Pubmed, e utilizando palavras chaves: "Saúde mental AND população rural", determinantes de saúde mental AND população rural" e "iniquidades em saúde AND meio rural". Foram utilizados artigos publicados em português e poucos em inglês, para que houvesse uma comparação acerca da temática tanto no âmbito nacional quanto internacional, porém, tendo como foco de análise, o cenário nacional.

\section{RESULTADOS E DISCUSSÃO}

\subsection{A saúde mental da população rural e sua estreita relação com os} determinantes sociais

Com o advento da revolução industrial, o mundo moderno passou por um processo de reordenamento, porém, diferente do ambiente urbano, o espaço rural ficou bastante marginalizado, uma vez que não foram cogitadas políticas públicas para viabilizar e garantir à população rural, direitos básicos já garantidos à 
população urbana, como o acesso à educação, saúde, lazer dentre outros (MOREIRA, 2008). Assim, as características que marcam bastante a vida em áreas rurais são a pobreza, condições de trabalho precárias, baixa escolaridade e escassez de recursos e serviços, principalmente na região nordeste (SAUER, 2010). Além disso, ao se analisar os indicadores socioeconômicos das áreas rurais, percebe-se que são bastante inferiores, se comparados com os indicadores das áreas urbanas. (IPEA, 2014).

As singularidades políticas, sociais e territoriais vivenciadas no âmbito rural associam-se a uma diversidade de fenômenos, como a destruição de florestas, erosão de terras, extinção de biodiversidade, fluxos migratórios dentre outros fatores. Essas questões compõem um quadro de vulnerabilidade ambiental e psicossocial, que impactam de forma bastante incisiva a saúde e a vida das populações rurais, que carecem de bens, recursos materiais e sociais e formas de se enfrentar esses fatores de risco (PINHEIRO et al., 2009). Além disso, as dificuldades de acesso a terra, os fatores ambientais e a pobreza têm trazido consequências psicossociais a essa população. Assim, viver em uma múltipla privação de serviços e bens causam impactos bastante negativos na saúde mental dessa população (SILVA; SANTANA, 2012).

O cuidado em Saúde Mental além de ser um tema bastante complexo e multideterminado ele envolve uma variedade de aportes tanto teórico quanto metodológicos, que por sua vez, implicam em posicionamento éticos e políticos. Desse modo, são levantados vários desafios teóricos e metodológicos na atenção em saúde e na gestão em saúde mental no Sistema Único de Saúde (SUS), ao se refletir a respeito do cuidado mental observando as condições de vida e saúde dessas populações rurais, bem como as relações cotidianas, as práticas e saberes tradicionais dessas populações (CIRILO; DIMENSTEIN, 2017).

Embora ao longo do tempo no Brasil, as políticas públicas de saúde buscaram abranger as particularidades e diversidades socioeconômicas, ambientais, culturais e de gênero da população brasileira (BRASIL, 1990), ainda há uma 
carência de atenção bastante significativa em relação à saúde das populações rurais (VIEIRA, 2010).

Diante desse cenário, observa-se que nessas populações rurais a vulnerabilidade social é uma realidade cujos impactos na saúde são bem visíveis, como as doenças associadas a discriminação, sobrecarga de trabalho, exploração, desvalorização opressão e violência, questões essas destacadas por diversos pesquisadores. Além disso, uma carga de trabalho excessivo, a falta de lazer e ausência da participação, de conhecimento inter ambiente e comunicação, são questões que estão na base de várias doenças e agravos que acometem a população rural como, uso indiscriminado de medicamentos, alcoolismo doenças psicossomáticas, suicídio, transtornos psiquiátricos menores e "doenças dos nervos" (VELÔSO et al., 2016).

Ao se tratar das populações rurais, percebe-se que a vulnerabilidade psicossocial relacionada à pobreza, se agrava. Portanto a pobreza, a desigualdade e a enfermidade constituem um movimento típico de retroalimentação (COTTA et al., 2007). Um fator que além de ser decisivo, tem rebatimentos inequívocos na saúde mental é a pobreza principalmente, em relação ao uso abusivo de álcool e a prevalência de transtornos mentais comuns. Além disso, a pobreza torna aguda às iniquidades de saúde e sociais, acirrando também os processos de exclusão social dessas populações (DIMENSTEIN et al., 2017).

Segundo a Organização Mundial de Saúde (OMS, 2010) às situações que envolvem vulnerabilidade podem causar consequências psicossociais, assim como a marginalização e o estigma podem trazer impactos na autoconfiança, autoestima, além de diminuir a motivação e também interferir inclusive em projetos de vida. $A$ exposição cotidiana a abusos e violências pode ocasionar uso de psicoativos, gerar sintomas psicossomáticos e até causar doenças mentais graves. A saúde mental também é afetada quando há violação de direitos político-culturais, sociais e civis ou quando ocorre exclusão de oportunidades de educação e geração de renda para grupos sociais. Essas questões trazem implicações particulares à população rural, que historicamente sofre com as situações de pobreza. Tem-se também como 
agravante desse cenário de vulnerabilidades, o fato de não haverem soluções e respostas dadas pelos sistemas de saúde, aos problemas comumente vivenciados por essa população. Observa-se, portanto, que a população rural muitas vezes a vítima do esquecimento dos governantes e apresenta grande dificuldade para acessar serviços básicos de saúde e de alta complexidade (BARBOSA; GUEDES; SILVA, 2017).

Conclui-se que além de menos visíveis, esses grupos são mais afetados e mais difíceis de serem contemplados por políticas públicas e investigadores. Essas condições configuram uma desvantagem social que por sua vez, aumentam não apenas a vulnerabilidade social, mas também o sofrimento psíquico, com ênfase ao desenvolvimento de transtornos mentais comuns (TMC) e do uso abusivo de álcool. Diversos estudos também reforçam a interligação entre as condições de vida mais precarizadas e a saúde mental, englobando elementos como as questões de gênero, a violência e as modalidade de apoio social, por fazerem parte do cotidiano dessa população (COSTA et al., 2014; LOUREIRO et al., 2014; MOURA; XIMENES; SARRIEIRA, 2014; REMOALDO; NOGUEIRA, 2013; SILVA; MENEZES, 2013).

Uma evidência que não se restringe apenas ao nível nacional é escassez de estudos sobre intervenções em contextos rurais e a prevalência de transtornos mentais nessas populações. As publicações que abordam a morbidade psiquiatra em zonas rurais referem-se geralmente, a uma prevalência alta de transtornos mentais ao compará-los com os índices desses mesmos acometimentos em áreas urbanas, enfatizando a necessidade da adoção de políticas específicas para essas populações e desse modo reforçando também, os princípios que norteiam SUS (VELÔSO et al., 2016).

No âmbito internacional, também são poucos os estudos realizados a respeito da saúde mental da população rural (MINISTÉRIO DE SANIDAD Y POLÍTICA SOCIAL, 2011). No Brasil um dos poucos estudos sobre esse tema, o relatório feito pela Confederação Nacional dos Trabalhadores de agricultura, (CONTAG, 2013), observou que os agravos mais frequentes como as constantes dores de cabeça, hipertensão, problemas de coluna, disfunções gastrointestinais, insônia, alergias e 
problemas de pele são problemas cuja base do sofrimento pode ter uma origem psicossocial, relacionada às condições de trabalho e vida em contextos rurais.

De acordo com pesquisas nacionais, há muitos fatores relacionados à alta morbidade psiquiátrica em áreas rurais, dentre eles estão: a exposição a inseticidas, pesticidas, agrotóxicos e adubos, sem a devida proteção; cargas de trabalho diárias bastante pesadas, além das más condições de escolaridade e de uma estrutura de cunho tecnológico para a produção (FARIA et al., 1999; FARIA et al., 2000; LEVIGARD; ROZEMBERG, 2004; SANTOS, J.C.B, 2011; FONTOURA, JÚNIOR et al., 2011). Inclusive a migração de muitos jovens as áreas urbanas, pode também ter contribuído no aumento do índice de prevalência da morbidade psiquiátrica (FARIA et al., 1999).

Embora haja uma relativa exiguidade de investigações acerca da saúde mental da população rural, alguns estudos mostraram uma maior suscetibilidade dessa população ao uso abusivo de tabaco e álcool (GOMES 2003; OLIVEIRA, 2012) e também, para o desenvolvimento de transtornos mentais comuns (TMC), que são quadros sintomáticos, porém não psicóticos e sem uma patologia orgânica associada, como a fadiga, a irritabilidade, a insônia o esquecimento e dificuldade de concentração (COSTA; LUDEMIR, 2005; FARIA et al., 2000). Através de estudos epidemiológicos, observou-se, por exemplo, uma relação existente entre TMC e as variáveis relacionadas às condições de vida e estrutura ocupacional, onde há uma prevalência alta dessa doença pessoas que vivem em situações de maior vulnerabilidade psicossocial e econômica (LUDEMIR; FILHO, 2002). Ao associar fatores como gênero feminino, baixa escolaridade, a condição precária de trabalho e pobreza, o risco de desenvolvimento de TMC aumenta, de modo que diversos estudos epidemiológicos realizados em áreas urbanas e rurais expressam uma maior prevalência dessa doença no sexo feminino (LUDEMIR, 2000; SILVA; SANTANA, 2012).

Analisando de um modo particular alguns contextos rurais, a forte influência dos determinantes sociais da saúde é perceptível de uma forma bastante nítida, como na região nordeste, onde a seca causa muitas consequências psicossociais 
que por sua vez, tendem a gerar insegurança em relação ao futuro ou mesmo, sentimento de tristeza, desânimo desesperança e fatalismo, que causam impacto no processo de saúde e modo de vida da população à medida que vão influenciando e remoldando os ideais que formam sobre o mundo e sobre si e também as estratégias meios de enfrentarem as adversidades (CAMURÇA et al., 2016). Além disso, Com o advento da crise financeira, são agravadas as situações de pobreza, desemprego, e endividamento até em populações rurais, fatores esses que quando associados, contribuem para o agravamento de transtornos mentais, como ansiedade, depressão, suicídio, uso de álcool e outras drogas, além de contribuir para o aparecimento de sofrimento psíquico (ANTUNES, 2015; SILVA et al., 2015).

Em um estudo, realizado com moradores de assentamentos rurais, foi constatado que os riscos socioeconômicos e ambientais quando associados, podem afetar as condições de vida e gerar impactos na qualidade de vida das pessoas, desencadeando transtornos mentais comuns e bastante sofrimento para elas. Notou-se também, que no âmbito rural, existe um conjunto de estressores e características que estão associados aos problemas de saúde mental, sendo que para a resolução desses agravos, faz-se necessário uma articulação de diversos fatores presentes nos territórios que ocasionam diferentes experiências de sofrimento. Assim, percebe-se que o cuidado em saúde mental deve ser compreendido a partir das dinâmicas e particularidades socioterritoriais, das condições de trabalho, de vida e dos determinantes sociais de saúde da população rural (COSTA; DIMENSTEIN; LEITE, 2014).

Em outro estudo realizado com Trabalhadores da produção Canavieira de Ribeirão Preto, foi observado que muitos deles apresentavam padrões bem altos de desgaste biopsíquico, estresse e alcoolismo, devido às alterações na agroindústria, algo que tem gerado exclusão social, não apenas a migração para áreas urbanas e também a pauperização do agricultor (ALESSI; NAVARRO, 1997). Observou-se também que no meio rural, houve um aumento do número de suicídios e consumo de álcool entre homens e mulheres. A violência, principalmente com as mulheres, é algo que vem sendo investigado, e por ser bastante velado e invisível, só aparece 
nos serviços de saúde em forma de depressão, estresse, ansiedade e queixas difusas (LEVIGARD; ROZEMBERG, 2004). A violência produz sérias consequências à saúde física e mental de mulheres e homens residentes de áreas rurais (VELÔSO et al., 2016).

Percebe-se também que o modelo socioeconômico vigente vem produzindo custos sociais que causam bastante impacto na vida dessa população. As condições de trabalho estão conformando um cenário epidemiológico bastante preocupante no meio rural, onde há uma prevalência alta de morbidades relacionadas ao sistema osteomuscular, bem como transtornos mentais dentre outras doenças, devido à inadequação dos equipamentos de proteção individual (EPI) e ao crescente uso de insumos químicos, que ocasionam acidentes e processos graves de intoxicação. As condições precárias de vida da População do Campo e Floresta também se relacionam com agravos de saúde como diarreias, parasitoses intestinais, resultante da falta de acesso à rede hídrica e de saneamento básico; com doenças infectocontagiosas como leishmaniose e Chagas e com elevado índice de mortalidade infantil (MINISTÉRIO DA SAÚDE, 2011).

\subsection{As iniquidades em saúde e sua influência na saúde mental da população rural}

Apesar dos avanços promovidos pela reforma psiquiátrica no Brasil, que favoreceu uma expansão na rede de cuidados em saúde mental, ainda observa-se uma necessidade intrínseca de ampliar esses serviços e de levar esse modelo de atenção também aos contextos rurais (VELÔSO et al., 2016). Além disso, houve um crescimento da Rede de Atenção Psicossocial (RAPS), especialmente dos Centros de Atenção Psicossocial, (CAPS) no país como um todo. Contudo, a população rural vive uma realidade onde a atenção em saúde mental em zonas rurais ainda se caracteriza como um problema. Isso porque o acesso a esses serviços é bastante difícil, e o modo como é feito a dinâmica organizacional deles, não favorece nem oportuniza a participação da população à rede de atenção psicossocial (SILVA; 
DIMENSTEIN; LEITE, 2013). Esse fato demonstra o quanto às políticas públicas em saúde mental não tem dado uma devida atenção à população rural (RIBEIRO; MARTINS; OLIVEIRA, 2009).

Embora o Brasil tenha uma realidade essencialmente urbana, o sistema único de saúde também deve abranger as populações rurais. Para isso, se faz necessário que as práticas desse sistema de saúde sejam adequadas e adaptadas às necessidades e particularidades vivenciadas pela população rural, e que difere do modo de vida urbano. Diante disso percebe-se que a saúde no âmbito rural não deve ser pensada e executada com base em um modelo de saúde urbano. (BARBOSA; GUEDES; SILVA, 2017). É possível observar também, lacunas na efetivação de políticas públicas específicas e na assistência à saúde de forma direcionada às necessidades de mulheres e homens moradores de áreas rurais, que possuem maior dificuldade no acesso à serviços de saúde são mais vulneráveis ao adoecimento, devido a um déficit de autocuidado e maior exposição à riscos, muitas vezes associados às condições de trabalho e de vida (VIEIRA, 2010). Por conta disso, os moradores de áreas rurais sofrem com ausência em seu cotidiano, de políticas públicas, principalmente no âmbito da atenção primária e saúde mental (SILVA; DIMENSTEIN; LEITE, 2013).

Alguns importantes desafios encontrados na zona rural e que afetam a saúde mental são identificados nesses estudos, como a disponibilidade precária de serviços de saúde, sendo que os existentes não conseguem responder a problemas de saúde que ultrapassam o âmbito das doenças infectocontagiosas, algo que ocorre com as doenças de trabalho e as doenças inespecíficas já que os profissionais encontram-se despreparados para o atendimento desse tipo de demanda (SCHWARTZ, 2002). Dentre alguns desafios destaca-se o fato dos moradores percorrerem longas distâncias para conseguirem acessar os serviços de saúde e assistência especializada, o que por sua vez, estimula a automedicação. A dificuldade do desenvolvimento de outra atividade laboral além da agricultura, principalmente em períodos de estiagem, além da tendência de viver de modo 
isolado em locais com poucos eventos comunitários ou sociais, o que dificulta novas estratégias de apoio social e de sociabilidades (VELÔSO et al., 2016).

Em dezembro de 2011 foi publicada a Política Nacional de Saúde Integral das Populações do Campo, da Floresta e das Águas (PNSIPCF), que firmou o compromisso de garantir a essas populações o acesso uma saúde guiada pelos princípios do SUS. Essa política tem um caráter transversal e intersetorial com responsabilidades firmadas entre diferentes setores Ministério da Saúde e do governo (BRASIL, 2011; SOARES et al., 2015). Tem como objetivo a garantia do acesso a serviços de saúde de forma humanizada, resolutiva e primando pela qualidade, visando contribuir também para diminuição das vulnerabilidades de saúde das populações do campo e floresta, e para melhorias na qualidade de vida dos agricultores familiares, camponeses, trabalhadores rurais, acampados, assentados, assalariados e temporários, comunidades remanescentes de quilombos, populações indígenas, ribeirinhas, e aquelas que habitam ou utilizam reservas extrativistas. (BRASIL, 2011).

Contudo, apesar das melhorias de acesso, e mesmo depois da publicação dessa política, ainda há uma considerável limitação de acesso da população rural aos serviços de saúde e de forma adequada (ROCHA; GERHARDTH, 2007; SOARES et al., 2015). Ademais no âmbito do SUS, essas populações ainda enfrentam dificuldades para acessar aos serviços de saúde, devido à baixa cobertura da Estratégia de Saúde da Família (ESF), do Programa de Agentes Comunitários de Saúde (ACS), e também do Núcleo de Apoio à Saúde da Família (NASF) em suas localidades. Portanto, observa-se um déficit no que se refere à implantação dessas equipes de ESF (MACEDO et. al., 2016).

Cenários como esse desvelar que ainda há muita desigualdade de acesso principalmente em regiões rurais mais distantes e em áreas com menos investimentos e recursos em saúde, infraestrutura, educação, e geração de renda. Percebe-se também uma carência de políticas públicas voltadas aos territórios rurais e na prática da política, os atendimentos ainda são insuficientes. Há também pouco investimento governamental, ao se considerar a quantidade de famílias que estão 
inseridas em contextos rurais, e os profissionais além de confundirem as conjunturas, também comparam os serviços prestados em áreas urbanas com os de áreas rurais (BARBOSA; GUEDES; SILVA, 2017).

Observa-se também que não há atualmente uma política ou diretriz em vigor, voltada ao cuidado e atenção à saúde mental da população rural. Algo que acentua mais essa carência de atenção em saúde mental dessa população, é a dificuldade de se articular ações de cuidado nessa área ainda na atenção primária, assim como a falta de leitos e serviços especializados em saúde mental na RAPS das áreas rurais (COSTA; DIMENSTEIN; LEITE, 2014). Observa-se também, a existência de uma fragilidade em programas e políticas de saúde, por não haver uma efetiva articulação entre os serviços de saúde, algo que compromete e não garante a continuidade dos cuidados, tanto no nível individual quanto coletivo (SCHWARTZ, 2002).

Percebe-se que a discussão acerca da atenção à saúde ainda é bastante urbana e etnocêntrica, por não ser sensível às particularidades socioculturais dos demais contextos. Além disso, é no cotidiano das equipes de saúde que a lógica comunitária e territorial que norteia a atenção psicossocial necessita ser construída, para que haja mais efetividade nas respostas às necessidades na área de saúde mental das populações rural, que estão diretamente relacionadas com as iniquidades sociais que afligem população e a vida em áreas rurais (NETO; DIMENSTEIN, 2017).

Essas populações há muito tempo carecem do olhar das políticas públicas, em especial do sistema único de saúde brasileiro devido às desfavoráveis condições de saúde (SOARES ET. AL, 2015). Essas iniquidades afetam não somente a saúde física, mas também a saúde mental dessa população. É notória a necessidade de se ampliar as investigações acerca das populações rurais, para que esse conjunto imbricado e complexo de determinações seja passível de consideração e tenham uma maior atenção por parte dos gestores, formuladores de políticas públicas, e pelos trabalhadores que estão no cotidiano dos serviços de saúde, para que desse modo, seja possível ampliar o cuidado, o acompanhamento e o monitoramento dos 
casos, incluindo ações de promoção à saúde e prevenção, visando assim, enfrentar as iniquidades que interferem e condicionam a saúde mental (DIMENSTEIN et al., 2017).

A população rural apresenta determinantes sociais e índices de saúde cujo impacto é maior e mais devastador do que em populações urbanas. Além disso, alguns problemas de saúde, como doenças, exposições e acidentes ocorrem com mais frequência nas zonas rurais. Os índices de cobertura no âmbito da prevenção são bem piores nossas aéreas também. Para essa população, o acesso a serviços de saúde em todos os níveis, geralmente é mais difícil, assim como a longevidade ou integralidade do cuidado. Algo também observado tanto no Brasil quanto no mundo todo, é a falta de recursos humanos no âmbito rural (ANDO et al., 2011).

\section{CONSIDERAÇÕES FINAIS}

Observa-se que a população rural é vítima do esquecimento por parte de políticas públicas diversas inclusive na área da saúde. Esse esquecimento tem agravado ainda mais a situação de saúde dessa população que vive em situação de vulnerabilidade diversos fatores e particularidades relacionadas aos condicionantes e determinantes sociais e as iniquidades de saúde, que afetam a saúde física e psicossocial dessa população. Além disso, a questão da saúde mental é agravada por falta de estudos que demonstram a real situação de saúde mental dessa população. Assim, observa-se uma necessidade bastante urgente do desenvolvimento de políticas e estratégias voltadas à saúde mental dessa população, observando suas particularidades e especificidades. É necessário que haja uma seleção dos profissionais de saúde que tenham perfil para trabalhar com as populações rurais, além de uma capacitação para esses profissionais. Também se faz necessário o desenvolvimento de mais estudos que demonstram o real cenário relacionado aos transtornos mentais, o uso de álcool e outras drogas também na população das zonas rurais, de modo a subsidiar e contribuir para 
desvelar essas questões que são pouco evidenciadas e que deveriam ser analisadas com mais atenção e cuidado.

\section{REFERÊNCIAS BIBLIOGRÁFICAS}

ADLER, N. Behavioral and social sciences research contributions in NIH Conference on Understanding and Reducing Disparities in Health, 2006.

ALESSI, N. P.; NAVARRO, V. L. Saúde e trabalho rural: o caso dos trabalhadores da cultura canavieira na região de Ribeirão Preto, São Paulo, Brasil. Cadernos de Saúde Pública, v. 13, p. 111-121, 1997.

ALMEIDA FILHO, N. et al. Research on health inequalities in Latin America and the Caribbean: Bibliometric analysis (1971-2000) and descriptive content analysis (1971-1995). Am J Public Health, n. 93, p. 2.037-2.043, 2003.

ALVES, A. A. M.; RODRIGUES.; NUNO.; F. R. Determinantes sociais e económicos da Saúde Mental. Revista Portuguesa de Saúde Pública, v. 28, n. 2, p. 127-131, 2010.

ANDO, N. M. et al. DECLARAÇÃO DE BRASÍLIA" O Conceito de rural e o cuidado à saúde". Revista brasileira de medicina de família e comunidade, v. 6, n. 19, p. 142-144, 2011.

ANTUNES, J. A. P. J. Crise económica, saúde e doença. Psicologia, Saúde e Doenças, v. 16, n. 2, p. 267-277, 2015. Disponível em: http://www.scielo.mec.pt/pdf/psd/v16n2/v16n2a11.pdf. Acesso em: 8 nov. 2018

BARATA, R. B. Iniquidade e saúde: a determinação social do processo saúde-doença. Revista Usp, n. 51, p. 138-145, 2001. 
BARBOSA; A. C.; GUEDES, E.S.; SILVA, J. P. T. Acesso das comunidades rurais aos serviços da saúde da família no Brasil, 2017.

BRASIL, Casa Civil. Lei no 8.080, de 19 de setembro de 1990. Diário Oficial da união, v. 128, n. 182, 1990.

BRASIL. Política nacional de saúde integral das populações do campo e da floresta (PNSIPCF), Brasília, 2011. Disponível em: <http://bvsms.saude.gov.br/bvs/saudelegis/gm/2011/prt286602122011.html>.

Acesso em: 24 nov. 2018.

BUSS, P. M.; PELLEGRINI FILHO, A. A saúde e seus determinantes sociais. Physis: revista de saúde coletiva, v. 17, p. 77-93, 2007.

BUSS, P. M.; PELLEGRINI FILHO. As Iniquidades em saúde no Brasil, nossa mais grave doença: comentários sobre o documento de referência e os trabalhos da Comissão Nacional sobre Determinantes Sociais da Saúde. Cadernos de Saúde Pública, v. 22, p. 2005-2008, 2006.

CAMPOS, L.; SATURNO, P.; CARNEIRO, A. V. Plano Nacional de Saúde 2011-2016: a qualidade dos cuidados e dos serviços. Lisboa: Alto Comissário da Saúde, 2010.

CAMURÇA, C. E. S. et al. Implicações psicossociais da seca na vida de moradores de um município da zona rural do nordeste do Brasil. Avances en Psicología Latinoamericana, v. 34, n. 1, p. 117-128. 2016.

CARRAPATO, P.; CORREIA, P.; GARCIA, B. Determinante da saúde no Brasil: a procura da equidade na saúde. Saúde e Sociedade, v. 26, p. 676-689, 2017.

CASTELLANOS, P.L. Epidemiologia, saúde pública, situação de saúde e condições de vida. Considerações conceituais. In: Barata, R.B. (org.) Condições de vida e situação de saúde. Rio de Janeiro. ABRASCO, 1997.

CEBES, Centro Brasileiro de Estudos de Saúde. Avaliação da Atenção Básica à Saúde no Brasil. Saúde em Debate, 2014. Disponível 
em:<http://cebes.org.br/site/wp-

content/uploads/2014/11/RSD AB WEB 031114.pdf, $>$. Acesso em 29 out. 2018. 2001.

CHAUÍ, M. S. Escritos sobre a universidade. São Paulo. Editora UNESP,

CIRILO N.; LEITE, M.; DIMENSTEIN, M. Saúde Mental em Contextos Rurais: o Trabalho Psicossocial em Análise. Psicologia: Ciência e Profissão, v. 37, n. 2, p. 461-474, 2017.

COMISSÃO NACIONAL SOBRE DETERMINANTES SOCIAIS DA SAÚDE. As causas sociais das iniquidades em saúde no Brasil: Relatório Final da CNDSS. Editora Fiocruz, 2008.

COSTA, A. G.; LUDERMIR, A. B. Transtornos mentais comuns e apoio social: estudo em comunidade rural da Zona da Mata de Pernambuco, Brasil. Cadernos de Saúde Pública, v.21, n.1, p.73-79, 2005. Disponível em: <http://www.scielo.br/pdf/csp/v21n1/09.pdf>. Acesso em: 11 dez. 2018

COSTA, M. G. S. G.; DIMENSTEIN, M.; LEITE, J. F. Condições de vida, gênero e saúde mental entre trabalhadoras rurais assentadas. Estudos de Psicologia (Natal), 19(2), 145-154, 2014.

CONFEDERAÇÃO NACIONAL DOS TRABALHADORES DA AGRICULTURA. Relatório da "Escuta Itinerante: Acesso dos povos do Campo e da Floresta ao SUS. 2013. Disponível em: $<$ http://www.contag.org.br/index.php? modulo=portal\&acao=interna\&codpag=101\&id= 9668\&data=11/06/2014\&nw=1\&mt=1\&in=1>. Acesso em: 28 out. 2018.

COTTA, R. M. Mitre et al. "Pobreza, injustiça, e desigualdade social: repensando a formação de profissionais de saúde." Rev. bras. educ. med. [online]. 2007, vol.31, n.3, pp. 278-286, 2007. 
DIMENSTEIN, Leite, J.; Macedo, J. P.; Dantas, C. Condições de vida e saúde mental em contextos rurais. Serviço Social e Saúde, v. 16, n. 1, p. 151-158, 2017.

DIMENSTEIN, M.; Macedo, J. P. S.; Leite, J.; Dantas, C., da Silva, M. P. R. Iniquidades Sociais e Saúde Mental no Meio Rural. Psico-USF, v. 22, n. 3, p. 541553, 2017.

DIMENSTEIN, M et al. Condições de vida e saúde mental em assentamentos de reforma agrária no nordeste brasileiro. In P. Santana \& P. Nossa P. (Eds.), A Geografia da Saúde no cruzamento de saberes (pp. 113-116). Coimbra, Portugal: Grupo de Investigação em Geografia da Saúde. 2014.

DIMENSTEIN, M. et al. Determinação social da saúde mental: contribuições à psicologia no cuidado territorial. Arquivos Brasileiros de Psicologia, v. 69, n. 2, p. 72-87, $2017 . \quad$ Disponível em <http://pepsic.bvsalud.org/scielo.php?script=sci_arttext\&pid=S1809$52672017000200006 \&$ Ing=pt\&nrm=iso >. Acesso em 20 nov. 2018.

FARIA, N. M. X. et al. Processo de produção rural e saúde na serra gaúcha: um estudo descritivo. Caderno de Saúde Pública, v.16, n.1, p.115-128, 2000. Disponível em: <http://www.scielo.br/pdf/csp/v16n1/1570.pdf>. Acesso em: 18 nov. 2018.

FARIA, N. M. X. et al. Estudo transversal sobre saúde mental de agricultores da Serra Gaúcha (Brasil). Revista de Saúde Pública, v.33, n.4, p.391-400, 1999. Disponível em: <http://www.scielosp.org/pdf/rsp/v33n4/0467.pdf>. Acesso em: 18 out. 2018

FERREIRA, A. D. D. Processos e sentidos sociais do rural na contemporaneidade: indagação sobre algumas especificidades brasileiras. Estudos Sociedade e Agricultura, v.1, n. 18, p.28-46, 2002. Disponível em: <http://r1.ufrri.br/esa/art/200204-028-046.pdf>. Acesso em: 15 nov. 2018

FLORIANO, C. O. Identificação da qualidade de vida no meio rural no município de Major Vieira. Ágora: revista de divulgação científica, v. 16, n. 1, p. 99107, 2009. 
FONTOURA JÚNIOR, E. E. F. et al. Relações de saúde e trabalho em assentamento rural do movimento dos sem-terra na região de fronteira BrasilParaguai. Trabalho, Educação e Saúde, v. 9, n.3, p.379-397, 2011. Disponível em: <http://www.scielo.br/pdf/tes/v9n3/v9n3a03.pdf>. Acesso em: 20 dez. 2018.

GEORGE, F. Sobre determinantes da saúde. set 2011. Disponível em: <http://bit.ly/2vZqVke>. Acesso em: 5 nov. 2018

HELFAND, S. M.; ROCHA, R.; VINHAIS, H. E. Pobreza e desigualdade de renda no Brasil rural: Uma análise da queda recente. Pesquisa e Planejamento Econômico, 39(1), 59-80, 2009

KAWACHI, I. et al. Social capital, income inequality and mortality. Am J Public Health, n. 87, p. 1.491-1.498, 1997.

KESSLER, M. et al. Praticas de autocuidado de homens e mulheres do meio rural. Inova Saúde, v. 3, n. 2, p. 37-54, 2014

KRIEGER, N. A glossary for social epidemiology. Journal of Epidemiology and Community Health, London, v. 55, n. 10, p. 693-700, 2001.

LEVIGARD, Y. E.; ROZEMBERG, B. A interpretação dos profissionais de saúde acerca das queixas de nervos no meio rural: uma aproximação ao problema das intoxicações por agrotóxicos. Cadernos de Saúde Pública, v.20, n.6, p.15151524, 2004. Disponível em: <http://www.scielo.br/pdf/csp/v20n6/08.pdf>. Acesso em: 25 out. 2018.

LOUREIRO, A.; COSTA, C.; SANTANA, P. Determinantes contextuais da saúde mental. Condições de vida e saúde mental em contextos rurais, p. 73-93, 2016.

LOUREIRO, A. et al. Condicionantes da saúde mental e os instrumentos de avaliação de impactos. Em P. Santana (Eds.). Território e Saúde Mental em Tempos de Crise. (pp. 11-27). Coimbra, 2014.

LUDERMIR, A. B.; MELO, F. D. A. Condições de vida e estrutura ocupacional associada a transtornos mentais comuns. Revista de Saúde Pública, v. 36, p. 213221, 2002. 
MACEDO, J. P. et al . Condições de vida, pobreza e consumo de álcool em assentamentos rurais: desafios para atuação e formação profissional. Pesqui. prát. psicossociais, São João del-Rei , v. 11, n. 3, p. 552-569, dez. 2016 . Disponível em:<http://pepsic.bvsalud.org/scielo.php?script=sci_arttext\&pid=S1809-

$89082016000300003 \&$ Ing=pt\&nrm=iso $>$. acesso em: 19 out. 2019.

MALTA, D. C. et al. A Cobertura da Estratégia de Saúde da Família (ESF) no Brasil, segundo a Pesquisa Nacional de Saúde, 2013. Ciência \& Saúde Coletiva, $21(2), 327-338,2016$

MARMETINI, J. S. "Adoecimento mental em comunidades rurais do município de Centenário: perspectivas histórico-sociais." RELACult-Revista LatinoAmericana de Estudos em Cultura e Sociedade 3, no. 3, 2017.

MINISTERIO DE SANIDAD Y POLITICA SOCIAL. Rehabilitación psicosocial de pesonas con trastorno mental grave. Pautas de intervención en el domicilio y programas para contextos rurales. España, 2011.

MOREIRA F. Identidade cultural e cultura na fronteira homem/meio rural. Disponível em: <www.rizoma.ufsc.br/pdfs/765-of10c-st1.pdf>. Acesso em: 10 out. 2018.

MOURA, J. F; XIMENES, V. M., \& SARRIERA, J. C. A construção opressora da pobreza no Brasil e suas consequências no psiquismo. Quaderns de Psicología, 16(2), 85-93. 2014

NETO, M. C.; DIMENSTEIN, M. Saúde Mental em Contextos Rurais: o Trabalho Psicossocial em Análise. Psicologia: Ciência e Profissão, v. 37, n. 2, p. 461-474, 2017.

NORONHA, D. P.; FERREIRA, S. M. S. P. Revisões de Literatura. In: CAMPELLO, B. S.; CENDÓN, B. V. e KREMER, J. M. (Orgs.) Fontes de informação para pesquisadores e profissionais. Belo Horizonte: Ed. UFMG, p. 191, 2000. 
Oliveira D. L. L. C. A enfermagem e suas apostas no autocuidado: investimentos emancipatórios ou práticas de sujeição? Rev. Bras. de Enferm. 185188, 2012.

Organização Mundial da Saúde (OMS). Livro de recursos da Organização Mundial de Saúde sobre saúde mental, direitos humanos e legislação. 2005

Organização Mundial de Saúde (OMS). Integração de Saúde Mental nos cuidados de saúde primários: Uma perspectiva global. 2009

PACHECO A. E. Motivação para o autocuidado na atenção primária em saúde. Minas Gerais, 2012.

PATEL V, Kleinman A. Poverty and common mental disorders in developing countries. World Health Organization. 2003. Disponível em: http://www.ncbi.nlm.nih.gov/pmc/articles/PMC2572527/pdf/14576893.pdf. Acesso em 9 nov. 2018.

PELLEGRINI FILHO, A. Ciencia en pro de la Salud. Publicación científica y técnica no. 578. Washington DC: OPS/OMS, 2000.

PELLEGRINI FILHO, A. et al. Conferência mundial sobre determinantes sociais da saúde. Cadernos de Saúde Pública, Rio de Janeiro, v. 27, n. 11, p. 20802081, 2011.

PINHEIRO, T. M. et al. (2009). Saúde no campo. In A. Miranda (Coord.), Cadernos de textos da $1^{\text {a }}$ Conferência Nacional de Saúde Ambiental. p. 25-29 Brasília, DF: Associação Brasileira de Saúde Coletiva.2009.

PORTRAIT, F.; LINDEBOOM, M.; DEEG, D. Life expectancies in specific health states: results from a joint model of health status and mortality of older persons. Demography, New York, v. 38, n. 4, p. 525-536, 2001.

REMOALDO P.; NOGUEIRA, H. Variações e desigualdades socioterritoriais em saúde. Em P. Remoaldo \& H. Nogueira (Eds.), Desigualdades Socioterritoriais e Comportamentos em saúde (pp. 11-30) Lisboa, 2013. 
RIBEIRO, M.B.S.; MARTINS, S.T.F.; OLIvEIRA, L.R. Familiares de usuários vivenciando a transformação do modelo assistencial psiquiátrico. Estudos de Psicologia, v. 14, n. 2, p. 133-140, Natal, 2009.

ROCHA, L.; GERHARDT, T. Desnutrição e excesso de peso em crianças menores de cinco anos no meio rural de Arambaré, RS: (des) construindo ideias, repensando novos desafios. Ciência, Cuidado e Saúde, 206214. 2007.

ROSA C. R. Os determinantes sociais e o adoecimento mental. Rev. Eletrônica da rede de estudos do trabalho. 2007

SANTANA, P. Territórios e saúde mental em tempos de crise. Portugal. 2012. Disponível em: https://www.researchgate.net/publication/287216582.Acesso em: 23 nov. 2018

SANTOS, J. C. B. O movimento dos trabalhadores rurais sem-terra e as relações entre saúde, trabalho e ambiente em um assentamento rural no Estado do Rio de Janeiro, 2011. 1994.

SANTOS, M. Território globalização e fragmentação. São Paulo: Hucitec,

SAUER, S. Terra e modernidade: a reinvenção do campo brasileiro. São Paulo: Expressão Popular, 2010.

SCHWARTZ, E. O viver, o adoecer e o cuidar das famílias de uma comunidade rural do extremo sul do Brasil: uma perspectiva ecológica. 2002. Florianópolis, 2002.

SCOPINHO, R. A. Condições de vida e saúde do trabalhador em assentamento rural. Ciência \& Saúde Coletiva, Rio de Janeiro, v. 15, n. 1, p. 15751584 , 2010. Disponível em: $<$ http://www.scielo.br/scielo.php?script=sci_arttext\&pid=S141381232010000700069 >. Acesso em: 20 nov. 2018.

SILVA, D. F; SANTANA, P. R. Transtornos mentais e pobreza no Brasil: Uma revisão sistemática. Tempus - Actas de Saúde Coletiva, 175-185.2012. Disponível 
em: $\quad$.http://www.tempusactas.unb.br/index.php/tempus/article/view/1214/1099. Acesso em: 28 out. 2018.

SILVA, M. et al. A saúde mental e a crise econômica. In P. Santana. Territórios e saúde mental em tempos de crise (pp. 61-74). Portugal: Universidade de Coimbra. Disponível em em https://www.researchgate.net/publication/2872165822015. Acesso em: 17 nov. 2018

SILVA, R. A; MENEZES, J. A. Mulheres jovens e suas vivências com o uso do álcool no contexto das comunidades quilombolas. 2015. Disponível em: <http://www.ufpb.br/evento/lti/ocs/index.php/18redor/18redor/paper/viewFile/732/78> Acesso em: 29 out. 2018.

SILVA, V. H. F.; DIMENSTEIN, M.; LEITE, J. F. O cuidado em saúde mental em zonas rurais. Mental, v. 10, n. 19, p. 267-285, 2013.

SANTANA, P. Territórios e saúde mental em tempos de crise. Portugal: Universidade de Coimbra. 2015.2 Disponível em: https://www.researchgate.net/publication/287216582. Acesso em: 11 nov. 2018

SOARES, R.A. S et al. Determinantes socioambientais e saúde: O Brasil rural versus o Brasil urbano. Tempus Actas de Saúde Coletiva, v. 9, n. 2, p. 221-235, 2015.

SOUZA, M. A. A. Apresentação: Milton Santos, um revolucionário. OSAL: Observatório Social de América Latina. Año 6, n. 16. Buenos Aires: CLACSO, 2005. Disponível em: <http://bibliotecavirtual.clacso.org.ar/ar/libros/osal/osal16/D16Santos.pdf >.Acesso em: 13 nov. 2018.

TARLOV, A. Social Determinants of Health: the sociobiological translation. In: BLANE, D.; BRUNNER,E.; WILKINSON, R. (Eds.). Health and Social Organization. London, p. 71-93, 1996.

TRAVASSOS, C.; VIACAVA, F. Acesso e uso de serviços de saúde em idosos residentes em áreas rurais, Brasil, 1998 a 2003 . 2007. 
VALADARES, Alexandre Arbex. O gigante invisível: território e população rural para além das convenções oficiais, 2014.

VELÔSO, THELMA MARIA GRISI. Saúde mental: saberes e fazeres. 21. ed. Campina Grande: eduepb, 2016.

VIEIRA, E. W. R. Acesso e utilização dos serviços de saúde de atenção primária em população rural do município de Jequetinhonha, Minas Gerais. Belo Horizonte, 2010.

WHITEHEAD, M. The concepts and principles of equity and health. International Journal of Health Services, Los Angeles, v. 22, n. 3, p. 429-445, 1992.

WILKINSON, R. Unhealthy societies. New York: Routledge, 1997

WORLD HEALTH ORGANIZATION. A conceptual framework for action on the social determinants of health. Geneva, 2010.

WORLD HEALTH ORGANIZATION; Calouste Gulbenkian Foundation. Social Determinants of Mental Health. Geneva, 2014

WORLD HEALTH ORGANIZATION. Mental health and development: Targeting people with mental health conditions as a vulnerable group. 2010. Disponível em::http://apps.who.int/iris/bitstream/10665/44257/1/9789241563949 eng.pdf. Acesso em: 28 nov. 2018

WORLD HEALTH ORGANIZATION. The world health report 2001.Mental health: new understanding, new hope. Geneva, 2001. Disponível em http://www.who.int/whr/2001/en/whr01 en.pdf.Acesso em 21 out. 2018.

WORLD HEALTH ORGANIZATION. The Ottawa Charter for Health Promotion. First International Conference on Health Promotion, Ottawa, 1986. Disponível em: http://www.who.int/healthpromotion/conferences/previous/ottawa/en/ index.ht. Acesso em : 21 nov. 2018 
